\title{
New technologies promise safer sex for women
}

With nearly 7,000 people being infected with HIV every day, societies around the world need new techniques to keep sex safe. Currently, condoms provide the highest protection against HIV infection during intercourse. Although male condoms go back as far as at least 1,000 B.C. in ancient Egypt, researchers are still trying to improve them. For example, a newly developed spray-on approach provides a custom fit condom (although the drying time required for the latex will have to be shortened for the product to reach market). The first female condom, however, didn't reach the market until 1992; and female condoms have not been widely used, partly owing to their cost, which is usually ten times as much as a male condom. Here are three new approaches to improving HIV protection for women.

\section{A new PATH}

To increase the use of female condoms, they need to be more convenient. PATH - a Seattle-based, international health organization-is developing a condom that is packed in an easyto-insert capsule that dissolves, releasing the pouch to unfurl inside the vagina. In addition, four dots of absorbent foam on the pouch hold this condom securely in the vagina during use. Moreover, the PATH condom consists of a thin material that testers seem to like in terms of sensitivity, and testing of the material has shown it can block particles smaller than HIV. Currently, this condom is being manufactured in China, and the clinical studies needed for marketing there are planned for this fall, hopefully followed by targeted introduction in several markets. In addition, a phase 1 trial in the US found that this condom caused less vaginal irritation, was easier to handle and was less likely to slip than other female condoms (Contraception 78, 465-473; 2008).

\section{pH-powered mesh}

Typically, the vaginal $\mathrm{pH}$ hovers around $4-5$, but the introduction of semen raises that $\mathrm{pH}$ to 7 . Patrick Kiser and his colleagues at the University of Utah are hoping to capitalize on that change to create a vaginal gel that captures HIV particles before they infect the vaginal wall after intercourse. More specifically,

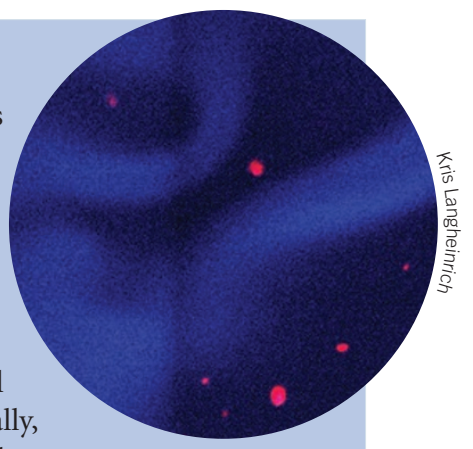
the researchers hope to use that $\mathrm{pH}$ change to transform a biocompatible polymer gel into a nanoscopic mesh. At $\mathrm{pH} 4-5$, this semisolid gel flows, coating the inner vaginal surface. When intercourse raises the $\mathrm{pH}$ to 7 , the gel turns more solid, as links between the polymers form a three-dimensional network with 30- to 50-nanometer openings-fine enough to ensnare 100-nanometer HIV particles (Adv. Funct. Mater. doi:10.1002/ adfm.200900757; 2009).

\section{Ring around the virus}

For ongoing HIV protection, women could eventually use a vaginal ring loaded with microbicides. That is the idea behind the BioRing. This ring, under development by Brij Saxena and his colleagues at Weill Medical College of Cornell University, is designed to be inserted in the vagina to deliver HIV-fighting drugs for up to 28 days. It might ultimately also deliver spermicidal agents to simultaneously provide contraception.

Using rings made from biosoluble acacia gum or nonbiodegradable hydrogels, BioRing provided effective drug delivery in vitro and in animal models. Using a range of anti-HIV compounds (loaded in the ring pictured on the left), the BioRing also blocked infection in human cell culture (AIDS 15, 917-922; 2009). Saxena and his colleagues are working on manufacturing BioRings suitable for clinical testing and are also seeking a pharmaceutical partner for further development. Mike May, Houston, Texas

\section{Growing pains plague children's health study}

The congressionally mandated National Children's Study (NCS), the largest long-term study of environmental and genetic effects on child health in the US to date, experienced some growing pains this month. A US Senate committee released a report on 4 August stating that it will reevaluate NCS funding after its 18 -month pilot phase finishes in 2010, raising questions about whether the full study will move forward as planned. There are reports that the cost of the study could balloon to $\$ 6$ billion, nearly twice what was first estimated. The Senate committee report accused the National Institutes of Health of a "serious breach of trust" for not relaying news of escalating costs sooner.

The NCS, which came about as part of the Children's Health Act of 2000, plans to follow 100,000 children from the womb to age 21 to tease out the causes of diseases such as asthma, obesity, diabetes and mental illness. Its first two pilot centers began recruiting subject this January, and five others followed in April.

Steven Hirschfeld, the acting director of the NCS since Peter Scheidt stepped down in July, told Nature Medicine that the new $\$ 6$ billion price tag represents a "fully loaded" version of the study, and that the protocols and scope could easily be scaled down to create a "core study that would still answer the important questions." Part of the goal of the ongoing pilot study, he says, is to determine exactly what should and should not be included in the expanded version.

Philip Landrigan, the principal investigator of the NCS pilot center at the Mount Sinai School of Medicine in New York, believes funding for the study will continue after the pilot phase. "I think it's our responsibility as scientists to educate the folks in Congress, and the public in general, about the costs and the benefits of the study," he says, and "the potential benefits are just extraordinary."

Melinda Wenner, New York 